\title{
The Diagnostic Value of Combined Detection of Kidney Injury Molecule-1 and Urine Microsomal Protein in Early Stage Nephropathy of Gestational Hypertension
}

HUA LI YIN, DONG LAN YUAN, XIN HUA BU, A. H. HUANG AND HUA QIAN*

Department of Obstetrics and Gynaecology, Hospital Affliated 5 to Nantong University (Taizhou People's Hospital), Taizhou, Jiangsu 225300, China

Yin et al.: Combined Detection in Early Stage Nephropathy of Gestational Hypertension

\begin{abstract}
To investigate the diagnostic value of urine kidney injury molecule-1 and urine microsomal protein combined test in early stage nephropathy of gestational hypertension. 72 patients with gestational hypertension treated at the hospital from December 2018 to December 2019 were randomly selected as subjects and 72 normal pregnant women who gave birth at the hospital during the same period were randomly selected as controls. Urinary microsomal protein levels were measured by immune enhanced turbidimetric assay, while urinary kidney injury molecule-1 levels were measured by enzyme linked immunosorbent assay. Blood urea nitrogen, high-sensitivity C-reactive protein levels, and specificity and sensitivity of urine kidney injury molecule-1 and urine microsomal serum proteins in the two groups and their subgroups were analyzed comparatively. The levels of urinary kidney injury molecule-1 and urinary microsomal serum protein were higher in the study group than in the control group. Among the three subgroups of the study group, urinary kidney injury molecule-1 and urinary microsomal serum protein levels were significantly increased in the mid and late pregnancy groups compared to the early pregnancy group. In the subgroup with impaired renal function, the urinary kidney injury molecule-1 and urinary microalbumin levels were significantly higher than those in the subgroup with normal renal function and the difference was statistically significant $(\mathbf{p}<\mathbf{0 . 0 5})$. In terms of blood urea nitrogen and high-sensitivity $C$-reactive protein levels, blood urea nitrogen and high-sensitivity $C$-reactive protein levels in the study group were higher than those in the healthy control group and estimated glomerular filtration rate was lower than those in the healthy control group. Urinary kidney injury molecule-1 level and urinary microsomal protein in the study group showed positive correlation with blood urea nitrogen and high-sensitivity C-reactive protein (both $\mathbf{p}<\mathbf{0 . 0 5}$ ), and negative correlation with Estimated glomerular filtration rate. In terms of sensitivity and specificity, the results showed that the specificity and sensitivity of the combined test were significantly higher than that of the individual test and the difference was statistically significant $(p<0.05)$. The detection of serum kidney injury molecule-1 and microsomal protein and other indicators to determine the degree of early renal impairment in pregnancy induced hypertension is beneficial for early clinical treatment and is of great clinical significance for pregnant women and pregnancy outcomes.
\end{abstract}

Key words: Gestational hypertension, kidney injury molecule-1, urinary microsomal protein, diagnostic value

Gestational hypertension is a common disease in clinical practice, which seriously threatens the life safety of pregnant women and perinatal babies. Patients may be asymptomatic in the early stage and can be found during physical examination. A few patients have headaches, dizziness, vertigo, palpitations and numbness of limbs and in the late stage, patients can appear server injury or lesions of heart, brain, kidney and other organs ${ }^{[1]}$. Long term hypertension can lead to renal glomerular hyperperfusion and glomerular arteriosclerosis and further affect the therapeutic effect and prognosis of

*Address for correspondence E-mail: qhtzrmyy@126.com patients with gestational hypertension ${ }^{[2,3]}$. When the hypertensives get to have proteinuria or increased blood creatinine levels, the damage of hypertension to kidney function is already severe and it's difficult to reverse the kidney injury for most treatments. However, if those indicators which can early reflect the changes in renal function are used to be detected when hypertension just occurs, the changes in renal function will be detected early and appropriate treatment can be given in time to avoid serious harm to mothers and babies. At present, the most commonly used detection index is serum creatinine, but in clinical it means patients with hypertension have had relative serious kidney 
impairment when serum creatinine becomes increased. Therefore, an indicator that can reflect renal impairment earlier than blood creatinine is needed. When kidney function is impaired, urine protein will appear in the urine ${ }^{[4-6]}$. Related studies at home and abroad have shown that the content of albumin in urine can help clinicians determine whether the renal tubules and glomeruli are damaged in patients with early stage of this disease. Urinary microalbumin can reflect renal injury sensitively, it suggests that renal impairment occurs when urine microalbumin hypertension is detected to reach $20 \mathrm{mg} / \mathrm{l}$ for patients with gestational, which has the value of early diagnosis for renal impairment in gestational hypertension. Kidney injury molecule- $1^{[7]}$ (KIM-1) is mainly present in kidney tissue and only a small amount of it is secreted under physiological conditions. Once the renal tubules are damaged, KIM1 can be induced to increased expression and released into the urine. Therefore, the level of KIM-1 can indirectly reflect the degree of renal tubular damage. At present, there is no relevant research report on the use of KIM-1 and urine microalbumin combined detection to evaluate renal impairment in gestational hypertension patients. This study randomly selected 72 patients with gestational hypertension and non-pregnancy and nonhypertensive patients who were treated in the hospital from December 2018 to December 2019, exploring and analyzing praticlal performance of co-detection of KIM-1 and urine microalbumin in early renal impairment for patients with gestational hypertension, providing a scientific basis for further reasonable and effective treatment of this disease. 72 patients with gestational hypertension who received treatment in the hospital from December 2018 to December 2019 were randomly selected as the research subjects and 72 normal pregnant women who received childbirth in the hospital during the same period were randomly selected as the control group. Research group: The age range is from 28 to $42 \mathrm{y}$, the average age is (29.5 \pm 3.9$)$ $\mathrm{y}$, the gestational age is 18 to $39 \mathrm{w}$, and the average gestational age is $(30.2 \pm 1.3)$ w. Education level: 24 in elementary school, 27 in middle school, 12 in high school and 9 in university. Among them, according to the gestational week, the study component can be divided into the first trimester group $(\mathrm{n}=21)$, the second trimester group $(\mathrm{n}=38)$ and the third trimester group $(n=13)$. Besides, if taking the glomerular filtration rate (GFR) as an indicator and combining the results of the nephrogram, the patients in each group were divided into two subgroups with normal renal function $(n=32)$ or impaired renal function $(n=40)$. Control group: The age range is 26 to $41 \mathrm{y}$, the average age is $(28.9 \pm 3.1) \mathrm{y}$, the gestational age is 19 to $38 \mathrm{w}$ and the average gestational age is $(30.6 \pm 1.0) \mathrm{w}$. Education level: 22 in elementary school, 28 in middle school, 11 in high school, 11 in university. All the selected pregnant women did not have liver or kidney disease, heart disease, diabetes, or essential hypertension before pregnancy. The pregnant women were all singleton pregnancies and did not take any drugs that might affect the test results during the investigation. All the pregnant women voluntarily participated in this test and signed the informed consent form. The research is approved by the medical ethics committee. There was no statistically significant difference between the study group and the control group in terms of age, gestational age, education level, etc., and they were comparable $(p>0.05)$. The content of urinary microalbumin is detected by immunoenhancement turbidimetric method. The specific detection steps are as follows: before going to bed, the nurse told the pregnant women in the study group and the control group to take $5 \mathrm{ml}$ of mid-morning urine for the next day, centrifuge for $10 \mathrm{~min}$ with a speed of $3000 \mathrm{r} / \mathrm{min}$, absorb the supernatant, the change of absorbance was detected at $630 \mathrm{~nm}$ wavelength and the detection kit was from sigma company. The level of urine KIM-1 was detected by enzyme-linked immunosorbent assay (ELISA). The kit was purchased from Saixintong (Shanghai) Biological Reagent Co., LTD. The detection operation was strictly in accordance with the kit instructions. Tecan InfinitePro full-wavelength multifunctional microplate reader is to measure serum Blood urea nitrogen (BUN) and high-sensitivity C-reactive protein (hsCRP) levels. Determination of renal injury: Estimated glomerular filtration rate $(\mathrm{eGFR}) \geq 60 \mathrm{ml} /\left(\mathrm{min}^{*} 1.73 \mathrm{~m}^{2}\right)$ indicated no renal injury, while eGFR $<60 \mathrm{~mL} /\left(\mathrm{min}^{*} 1.73 \mathrm{~m}^{2}\right)$ indicated the presence of renal injury. The calculation formula of eGFR was: $\mathrm{eGFR}=\mathrm{Ccr} \times 0.84 \times 1.73$ / BSA, while Ccr was creatinine clearance rate and body surface area (BSA), $\operatorname{Ccr}(\mathrm{min} / \mathrm{mL})=[(140$ age $) \times$ weight $\quad(\mathrm{Kg}) \times(0.85 \quad$ female $)] /(72 \times \mathrm{Ccr})$; $\operatorname{BSA}\left(\mathrm{m}^{2}\right)=0.007184 \times$ weight $\quad(\mathrm{kg}) \times 0.425 \times$ height $(\mathrm{cm}) \times 0.725$. The reference range of all indicators: urine microalbumin: $0 \sim 25 \mathrm{mg} / \mathrm{L}$, urine KIM-1:1 3mg/L. The data obtained in this study were statistically analyzed using SPSS 20.0 statistical software. Measurement data were expressed as mean \pm standard deviation $(\mathrm{x} \pm \mathrm{s})$, using $\mathrm{t}$ test; count data were expressed as percentage, using $\chi 2$ test. Test standard $\alpha$ is 0.05 and $p<0.05$ is considered as statistically significant. Correlation analysis adopts Pearson correlation; multivariate Logistic stepwise 
regression model is used to explore the risk factors of early renal injury in patients with pregnancy induced hypertension (HDP); receiver operating characteristic (ROC) curve is used to analyze the value of various indicators in predicting early renal impairment in patients with gestational hypertension. The area under the curve (AUC) of each indicator is compared by $\mathrm{Z}$ test. In terms of content of urine KIM-1 and urine microalbumin, pregnant women in the study group were higher than those in the control group and the difference between the two was statistically significant $(p<0.05)$, as shown in fig. 1 . In the three subgroups of the study group, compared with the first trimester group, the urine KIM-1 and urine microalbumin levels in the second trimester group and the third trimester group were significantly increased and the difference was statistically significant ( $<<0.05$ ), as shown in fig. 2 . In the subgroup of patients with impaired renal function,

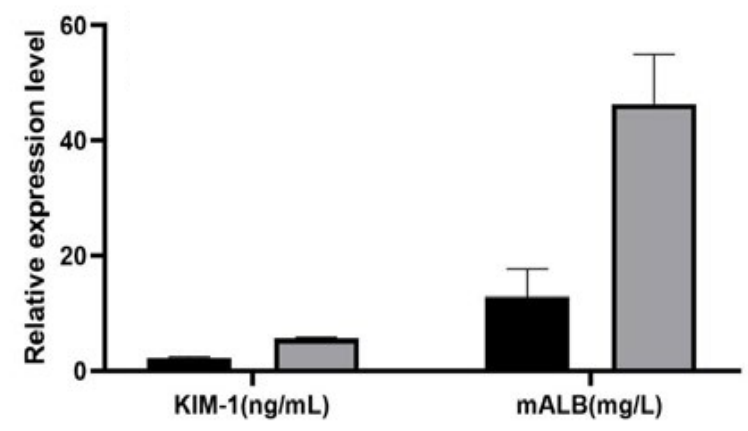

Fig. 1: The content of urine KIM-1 and urine microalbumin of pregnant women in the study group and the control group, Control ( $\square$ ), experimental ( $\square$ )
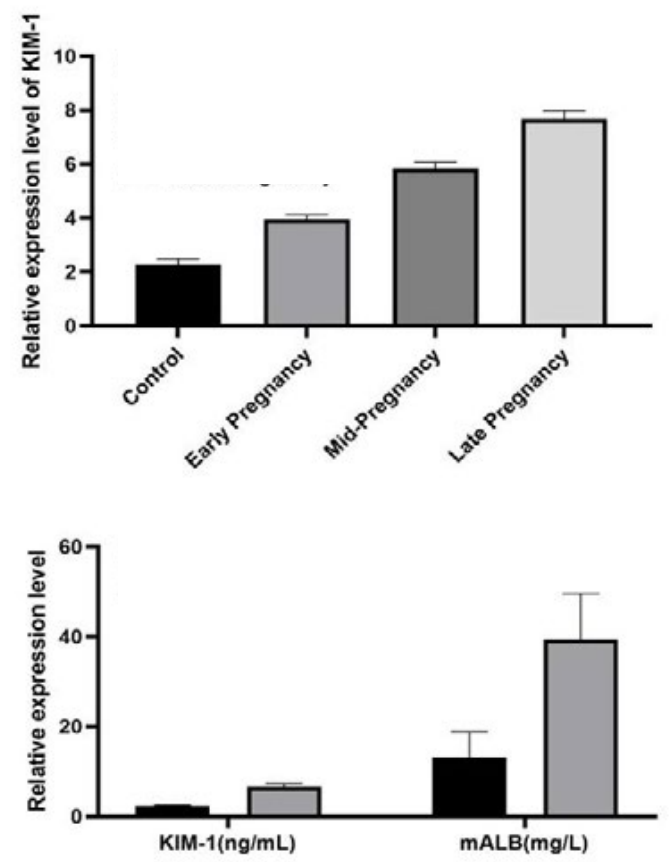

the contents of urine KIM-1 and urine microalbumin were significantly higher than those of the subgroup with normal renal function and the difference was statistically significant $(\mathrm{p}<0.05)$, as shown in fig. 2 .

The results showed that the BUN and hsCRP levels of the study group were higher than those of the healthy control group, and the eGFR was lower than that of the healthy control group $(\mathrm{P}<0.05)$. See Table 1 . The results showed that urine KIM-1 and urine microalbumin levels of the study group patients were positively correlated with BUN and hsCRP (both $\mathrm{p}<0.05$ ) and negatively correlated with eGFR, as shown in fig. 3 . The results showed that the specificity and sensitivity of combined tests were significantly higher than those of single tests, with statistically significant differences $(p<0.05)$, as shown in Table 2 . In clinical practice, HDP is a very common type of disease and its incidence tends to increase year by year ${ }^{[8]}$, which not only causes serious harm to pregnant women and perinatal babies, but also brings adverse effects on pregnancy outcomes ${ }^{[9]}$. During normal pregnancy, the pregnant woman's physiological and metabolic pressure increases with fetal development, which will increase blood coagulation, renal blood flow and glomerular filtration rate (GFR), etc. Both of these factors will aggravate the kidney's burden to varying degrees, leading to renal impairment in patients ${ }^{[10]}$. At present, the main clinical manifestations of HDP patients are gestational hypertension and pregnancy eclampsia. Pregnancy hypertension leads to increased systemic vascular pressure and pregnancy eclampsia

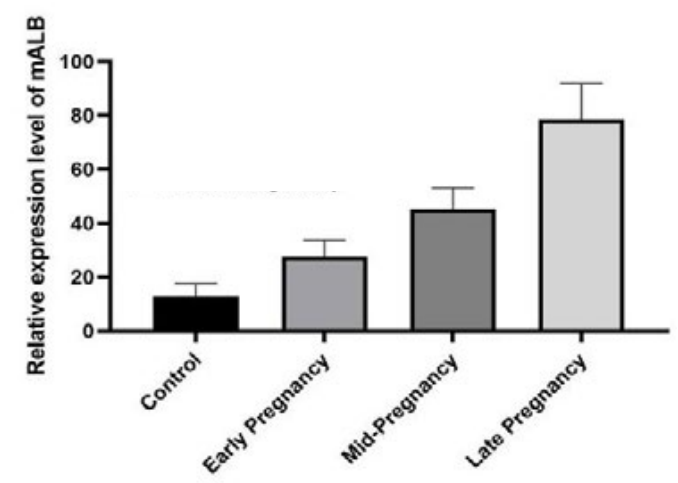

Fig. 2: The expression of KIM-1 and microalbumin in urine in several subgroups Renal health group ( $\square$ ), Renal impairment group ( $\square$ ) 
leads to systemic spasm of small blood vessels, resulting in reduced perfusion due to systemic organs being compressed by blood pressure. Decreased renal vascular perfusion is likely to lead to obstacles on the elimination of its metabolites, which can induce imbalances in electrolyte regulation and disorders of acid base balance. Therefore, early diagnosis of kidney injury caused by gestational hypertension is of great significance for reducing obstetric complications and improving the prognosis of patients. The microalbumin in human urine, with low molecular weight and negative charge, can freely pass through the glomerular filtration membrane of the kidney and is all reabsorbed into the proximal convoluted tubule of the kidney. Once renal lesions occur, the microalbumin in urine cannot be completely reabsorbed by proximal convoluted tubules and the filtration rate of glomeruli will decrease, resulting in the presence of protein in human urine, namely proteinuria ${ }^{[11-13]}$. When renal damage occurs, it may be that when the expression of protein marker routine

TABLE 1: COMPARISON OF BUN, EGFR AND HSCRP LEVELS BETWEEN THE TWO GROUPS

\begin{tabular}{lccc}
\hline Group & BUN & hsCRP & eGFR \\
\hline Research & $4.17 \pm 0.63$ & $120.59 \pm 10.33$ & $330.26 \pm 10.40$ \\
Control & $5.96 \pm 0.47$ & $81.28 \pm 12.46$ & $373.44 \pm 14.29$ \\
$\mathrm{p}$ & $<0.05$ & $<0.05$ & $<0.05$ \\
\hline
\end{tabular}

TABLE 2: COMPARISON OF THE SENSITIVITY AND SPECIFICITY OF KIM-1 AND MICROALBUMIN IN URINE

\begin{tabular}{|c|c|c|c|}
\hline Marker & $\operatorname{AUC}(95 \% \mathrm{Cl})$ & \multicolumn{2}{|c|}{ Sensitivity Specificity } \\
\hline KIM- 1 & $0.824(0.787 \sim 0.909)$ & 0.779 & 0.753 \\
\hline mALB & $0.836(0.712 \sim 0.936)$ & 0.764 & 0.771 \\
\hline KIM-+mALB & $0.897(0.787 \sim 0.958)$ & 0.914 & 0.937 \\
\hline
\end{tabular}

urinalysis is negative clinically, but the microalbumin can be expressed positive sensitively. In this study, we found that the content of microalbumin in the urine of the study group was significantly higher than that of the control group, indicating that renal lesions occurred in pregnant women, which may due to the damage on the glomeruli or tubules, or due to the obstruction of glomerular filtration rate caused by renal artery spasm. KIM-1 is a type I transmembrane protein that exists in the proximal tubules of the kidney ${ }^{[14]}$. Under normal circumstances, the kidney tissue hardly expresses KIM1. When the kidney is damaged, KIM-1 can be released in large quantities and enter the urine. Therefore, KIM1 in urine can be detected to indirectly assess the time and extent of kidney impairment. The latest research found $^{[15]}$ that KIM-1 can also be used as a functional molecule, the expression of it can be activated when the renal tubules are ischemia and hypoxia, mediating a series of signaling pathways to participate in renal injury repairmen. In this study, it was found that the content of KIM-1 in urine of the study group was significantly higher than that of the control group and the difference between the two was significant, suggesting that KIM1 can correctly indicate the symptoms of kidney injury. In addition, according to related research reports, KIM1 can be detected in urine within $2 \mathrm{~h}$ after the kidney injury occurs and there is no KIM-1 expression in tissues outside the kidney, which is an ideal marker for the diagnosis of kidney injury. $\mathrm{Cr}$, BUN, and eGFR are common important indicators that reflect kidney injury, while hsCRP is a sign of the severity of inflammation in the body and can reflect the severity of kidney injury to a certain extent. In this study, the levels of BUN and hsCRP in the pregnancy induced hypertension group
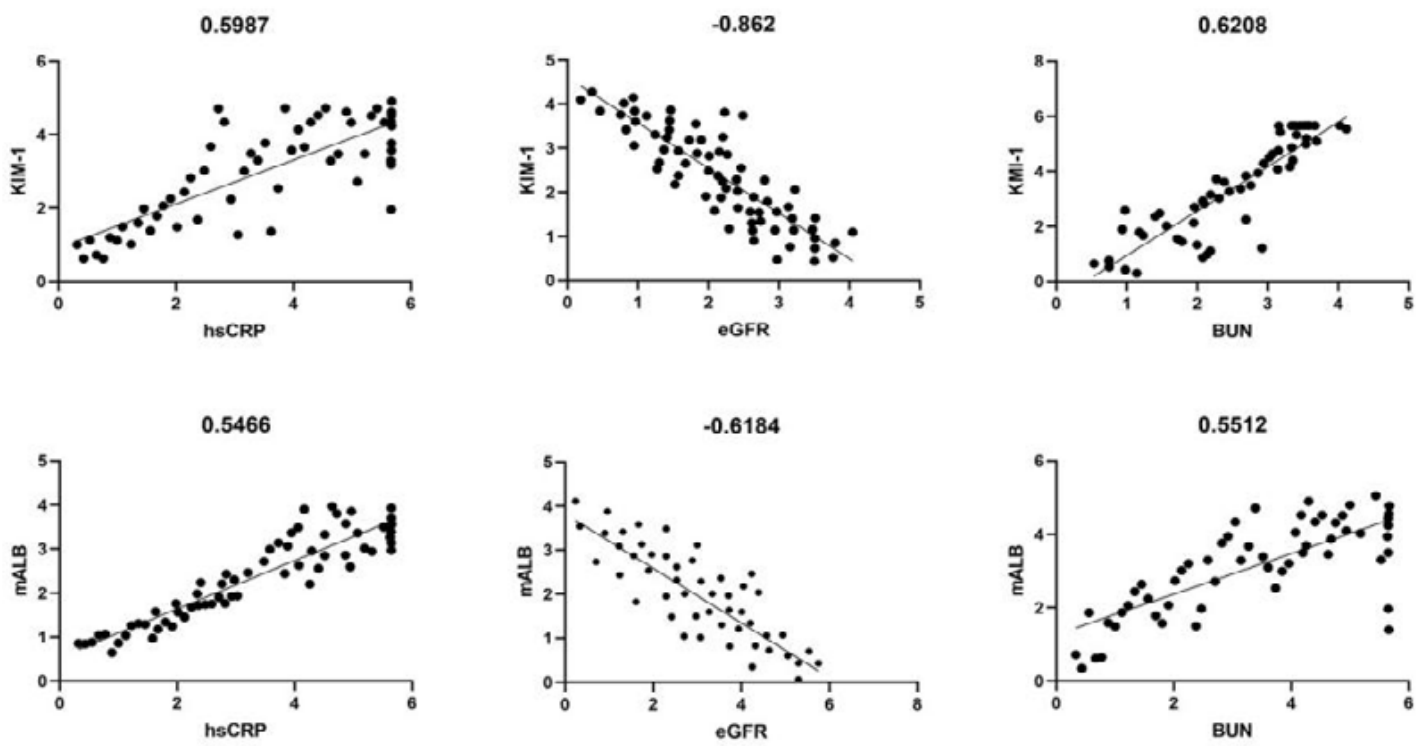

Fig. 3: Correlation between levels of urine KIM-1, microalbumin and BUN, eGFR, hsCRP of patients in the study group 
were higher than those in the healthy control group and the eGFR was lower than those in the healthy control group. The urine KIM-1 levels and urine microalbumin in the study group were positively correlated with BUN and hsCRP (both $\mathrm{p}<0.05$ ), negatively correlated with eGFR, suggesting that urine KIM-1 and microalbumin can indirectly reflect the levels of $\mathrm{Cr}, \mathrm{BUN}$ and eGFR. Due to the high stability of urine KIM-1 and specimen acquisition is easier than blood, urine specimens can be frequently retained for continuous detection when necessary. The results of this study also showed that the specificity and sensitivity of urine microalbumin of hypertensive patients in pregnancy in the study group were as high as $77.9 \%$ and $75.3 \%$, which is close to that of urine microalbumin from normal pregnant patients and the difference was not statistically significant. It is particularly noteworthy that the study also found that the specificity and sensitivity of KIM-1 level combined with urinary albumin to detect renal impairment in gestational hypertension were up to $91.4 \%$ and 93.7 $\%$, significantly higher than that of any single indicator, with obvious clinical significance. Compared with the single use of KIM-1 and trace albumin, combined detection has a great advantage. The joint of KIM-1 and microalbumin is very necessary for early detection, which can dynamically monitor renal impairment in patients with gestational hypertension and has important application value. In conclusion, the detection of urine KIM-1 and microalbumin to determine the extent of renal damage in the early stage of HDP is conducive to early clinical treatment and has very important clinical importance for pregnant women and pregnancy outcomes.

\section{Acknowledgements}

This work was supported by The Fifth Affiliated Hospital of Nantong University (Taizhou People's Hospital).

\section{Conflict of interests}

The authors declared no conflict of interest.

\section{REFERENCES}

1. Stevens LA, Coresh J, Schmid CH, Feldman HI, Froissart M, Kusek J, et al. Estimating GFR using serum cystatin $\mathrm{C}$ alone and in combination with serum creatinine: a pooled analysis of 3,418 individuals with CKD. Am J Kidney Dis 2008;51:395406.

2. Olaya-C M, Garrido M, Hernandez-Losa J, Sesé M, AyalaRamirez P, Somoza R, et al. The umbilical cord, preeclampsia and the VEGF family. Int J Womens Health 2018;10:783-95.

3. Blaufox MD, Aurell M, Bubeck B, Fommei E, Piepsz A, Russell C, et al. Report of the Radionuclides in Nephrourology
Committee on renal clearance. J Nucl Med 1996;37:1883-8.

4. Myers GL, Miller WG, Coresh J, Fleming J, Greenberg N, Greene $\mathrm{T}$, et al. Recommendations for improving serum creatinine measurement: a report from the Laboratory Working Group of the National Kidney Disease Education Program. Clin Chem 2006;52:5-18.

5. Trimarchi H, Muryan A, Martino D, Toscano A, Iriarte R, Campolo-Girard V, et al. Creatinine-vs. cystatin C-based equations compared with $99 \mathrm{mTcDTPA}$ scintigraphy to assess glomerular filtration rate in chronic kidney disease. J Nephrol 2012;25:1003.

6. Inker LA, Schmid CH, Tighiouart H, Eckfeldt JH, Feldman HI, Greene T, et al. Estimating glomerular filtration rate from serum creatinine and cystatin C. N Engl J Med 2012;367:20-9.

7. Peralta CA, Shlipak MG, Judd S, Cushman M, McClellan $\mathrm{W}$, Zakai NA, et al. Detection of chronic kidney disease with creatinine, cystatin $\mathrm{C}$, and urine albumin-to-creatinine ratio and association with progression to end-stage renal disease and mortality. JAMA 2011;305:1545-52.

8. Ireland $\mathrm{R}$. Use of urine albumin and cystatin $\mathrm{C}$ levels improves risk stratification in chronic kidney disease. Nat Rev Nephrol 2011;7:301.

9. Liang XL, Liu SX, Chen YH, Yan LJ, Li H, Xuan HJ, et al. Combination of urinary kidney injury molecule-1 and interleukin-18 as early biomarker for the diagnosis and progressive assessment of acute kidney injury following cardiopulmonary bypass surgery: a prospective nested casecontrol study. Biomarkers 2010;15:332-9.

10. Vaidya VS, Ozer JS, Dieterle F, Collings FB, Ramirez V, Troth $\mathrm{S}$, et al. Kidney injury molecule-1 outperforms traditional biomarkers of kidney injury in preclinical biomarker qualification studies. Nature Biotechnol 2010;28:478-85.

11. James M, Bouchard J, Ho J, Klarenbach S, LaFrance JP, Rigatto C, et al. Canadian Society of Nephrology commentary on the 2012 KDIGO clinical practice guideline for acute kidney injury. Am J Kidney Dis 2013;61:673-85.

12. Bellomo R, Ronco C, Kellum JA, Mehta RL, Palevsky P. Acute renal failure-definition, outcome measures, animal models, fluid therapy and information technology needs: the Second International Consensus Conference of the Acute Dialysis Quality Initiative (ADQI) Group. Crit Care 2004;8:1-9.

13. Verbrugge FH, Dupont M, Shao Z, Shrestha K, Singh D, Finucan $\mathrm{M}$, et al. Novel urinary biomarkers in detecting acute kidney injury, persistent renal impairment, and allcause mortality following decongestive therapy in acute decompensated heart failure. J Card Fail 2013;19:621-8.

14. Harel Z, Wald R, Bargman JM, Mamdani M, Etchells E, Garg AX, et al. Nephrologist follow-up improves all-cause mortality of severe acute kidney injury survivors. Kidney Int 2013;83:901-8.

15. Parikh CR, Han G. Variation in performance of kidney injury biomarkers due to cause of acute kidney injury. Am J Kidney Dis 2013;62:1023-6.

This is an open access article distributed under the terms of the Creative Commons Attribution-NonCommercial-ShareAlike 3.0 License, which allows others to remix, tweak, and build upon the work non-commercially, as long as the author is credited and the new creations are licensed under the identical terms

This article was originally published in a special issue, "Clinical Research in Pharmaceutical and Biomedical Sciences" Indian J Pharm Sci 2021:83(1)Spl issue1;97-101 